\title{
AUV navigation and guidance in a moving acoustic network
}

\author{
Aníbal Matos and Nuno Cruz \\ Faculdade de Engenharia da Universidade do Porto \\ Instituto de Sistemas e Robótica - Porto \\ Rua Dr. Roberto Frias, 4200-465 Porto \\ Portugal \\ \{anibal,nacruz\}@fe.up.pt
}

\begin{abstract}
This paper addresses the navigation and guidance of an AUV operating in a network of moving acoustic beacons. In this network, the vehicle position is obtained from acoustic signals exchanged between the vehicle and the beacons and is computed in a reference frame associated with the moving beacons. The paper describes the operation of the acoustic network, the mission specification and guidance system that computes the references for the vehicle controllers, and the navigation system that produces all the data required by the guidance system.
\end{abstract}

\section{INTRODUCTION}

This paper addresses the navigation and guidance of the Isurus AUV operating in a moving acoustic network. Traditionally, the beacons of an acoustic navigation network are moored and remain in the same place during the execution of one or several missions of the AUV. The number and location of the beacons are selected taking into account the size of the operation area, the occlusion of beacons by natural features, and the logistics associated to their mooring. In some scenarios, one or more of these factors greatly increase operational costs, either by requiring a large number of beacons or by increasing the complexity of beacon deployment. In such cases it is clearly advantageous to use a network of moving beacons instead of fixed ones.

The acoustic navigation network considered here is composed by two moving beacons. These two beacons define at each instant in time a moving coordinate frame. To determine its position in this moving frame, the vehicle uses a spherical long baseline (LBL) positioning algorithm. Besides the signals exchanged between the vehicle and each beacon, additional signal exchanges are necessary for the vehicle to determine the distance between both beacons, required to compute its position in the moving frame. All these signal exchanges are thoroughly described in the paper.

Since the AUV does not have any communication link and does not know in real time the location of the beacons, all the mission specification has to be done with respect to the moving frame. Therefore, the vehicle guiding system, responsible for computing the heading reference for the vehicle control system, uses only moving frame coordinates and thus produces a heading reference with respect to this frame. The vehicle is only able to determine its heading with respect to an earth fixed frame, using a magnetic compass. The paper describes a
Kalman filter based procedure that fuses together the absolute heading of the vehicle, its velocity with respect to the water, and its distances to the beacons, in order to produce in real time an estimate of the orientation of the moving frame. This estimate allows for the determination of the vehicle heading with respect to the moving frame, as required by the guidance an control systems.

The paper starts with a description of the Isurus AUV and its navigation system. Then, in section IV, the concept of a moving network is introduced. Section V addresses the operation of the acoustic network, presenting the main differences with respect to a network of fixed beacons. Section VI describes the specification of missions as well as the vehicle guidance algorithm required for the operation in a moving network. Section VII presents the navigation algorithm that computes all the data required for the vehicle guidance. Finally, in section VIII, simulation results are presented.

\section{ISURUS AUV OPERATIONS}

Isurus (Fig. 1) is a REMUS class AUV, built by the Woods Hole Oceanographic Institution, MA, USA, in 1997. These vehicles are low cost, lightweight AUVs specially designed for coastal waters monitoring [1]. The reduced weight and dimensions makes them extremely easy to handle, requiring no special equipment for launching and recovering. Isurus has a diameter of $20 \mathrm{~cm}$ and is about 1.5 meters long, weighting about $35 \mathrm{~kg}$ in air. Inside the hull, several subsystems have been improved or specifically developed at FEUP, contributing to the continuous enhancement of the vehicle performance and reliability. The maximum forward speed of the vehicle is 4 knots, however the best energy efficiency is achieved at about 2 knots. At this velocity, the energy provided by the rechargeable Lithium-Ion batteries lasts for over 20 hours (i.e., over 40 miles).

The first operational missions with Isurus took place in 1998, in the estuary of the river Minho, in the northern border between Portugal and Spain [2]. Since then, Isurus has been used in several oceanographic and environment monitoring missions. In the summer of 2002, on an innovative mission, the vehicle was employed on a monitoring plan for a sewage outfall, $3 \mathrm{~km}$ off the Portuguese coast, near Aveiro [3]. 


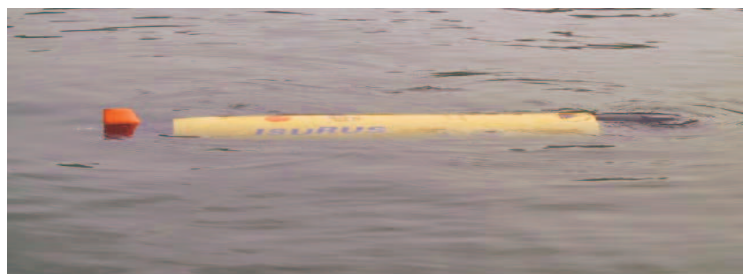

Fig. 1. The Isurus AUV.

Besides Isurus, tens of other Remus vehicles (manufactured by Hydroid, Inc. since 2001) are currently being operated around the world by university labs, oceanographic institutions, and navies. Main applications include hydrographic surveys, oceanographic studies and mine countermeasures. As recent examples of successful missions around the world, these vehicles have been used to map chemical plumes [4] and [5], and for mine countermeasures during Iraqi Freedom in the Arabian Gulf [6].

\section{ISURUS NAVIGATION SYSTEM}

Navigation accuracy is very important in almost every mission, but in the vast majority of applications standard LBL provides enough accuracy and the transponders may be easily deployed in the operation area. However, as new missions are envisaged [7], so there are new requirements as far as navigation is concerned.

FEUP has been working on underwater navigation systems in the last 6 years. This includes the development of underwater acoustic transponders [8] and integrated navigation systems both for ROVs and AUVs [9]. The position of the Isurus AUV is estimated in real time by fusing together data provided by several devices and systems. The vehicle depth is directly obtained by a depth pressure cell installed on the vehicle. The horizontal position is estimated by an extended Kalman filter, based on a simple model of the vehicle motion. This filter smooths the acoustic range measurements with dead reckoning data, composed by the vehicle attitude, obtained from a digital compass and a set of tilt sensors, and also by the vehicle velocity with respect to the water, obtained from an encoder that measures the propeller rotation speed. All the navigation data is logged by the vehicle onboard computer and is used after the mission completion to further improve the accuracy of the position estimates [10].

Usually, Isurus operates in an acoustic network composed by just 2 beacons located in different places. These are sufficient to determine the position of the vehicle, assuming it does not cross the line joining them (baseline).

The beacons used in the navigation network (fig. 2) are multi-frequency transponders and were developed at FEUP [8]. Each beacon can be configured to reply with a signal of a given frequency when interrogated by another signal of a different frequency. The frequencies used range from 20 to $30 \mathrm{kHz}$.

While navigating, the vehicle onboard software completely controls the exchange of acoustic signals and obtains the

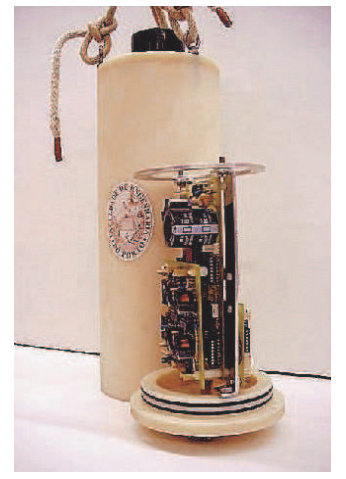

Fig. 2. FEUP acoustic beacon.

range measurements one at a time. To determine its range to a given beacon, the vehicle first sends an interrogation signal. All the beacons detect that signal but only the one identified with such interrogation signal will reply with a signal of a predefined frequency. Upon detection of the beacon reply, the vehicle measures the elapsed time, computes the range, and interrogates another beacon, starting a new range measurement.

The external tracking of the Isurus AUV can be accomplished just by listening to the acoustic signals exchanged between the vehicle and the navigation beacons and relies on a simple algorithm that was proposed in [8]. This simple tracking mechanism greatly enhances the operationality of the Isurus AUV, since the real time monitoring of the vehicle behavior allows for early detection of vehicle malfunctions that affect its trajectory [11].

\section{MOVING ACOUSTIC NETWORK}

Traditionally, the beacons of the acoustic navigation network are moored and remain in the same place during the execution of one or several missions. The number and location of the beacons are selected taking into account the dimensions of the operation area (to ensure enough signal power for detection and avoid adverse geometric configurations), the occlusion of beacons by natural features (to ensure straight line propagation), and the logistics associated to their mooring.

In some scenarios, one or more of these factors greatly affect operational costs, either by requiring a large number of beacons or by increasing the difficulty of beacon deployment. In such cases it is clearly advantageous to use a network of relocatable beacons instead of fixed ones.

One of these scenarios is the collection of CTD and bathymetric data in rivers with winding margins. For a typical mission, the vehicle has to cover an area as wide as the river and a few kilometers long, being impossible to cover the operation area with just a few fixed acoustic beacons.

For this type of mission, navigation can be assured by a moving acoustic network using two beacons. Each beacon will be attached to a small boat that will move slowly along the river, remaining close to a margin. The motion of the boats 


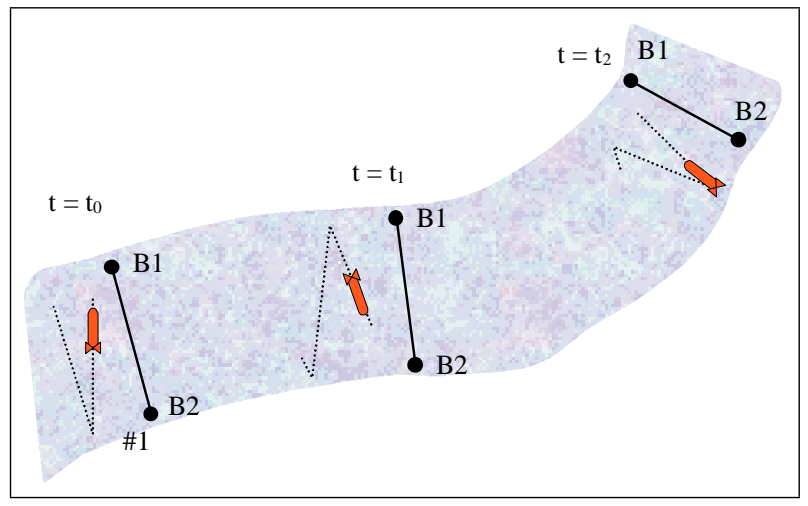

Fig. 3. Moving acoustic network.

will be coordinated such that both of them will continuously define a river cross section.

Since the navigation network is moving, the vehicle does not navigate with respect to an earth fixed frame, but navigates in the frame defined by the two navigation beacons. In this moving frame, the trajectory of the vehicle might be just a line, parallel to the baseline and at a given distance from it, and the vehicle just goes forth and back along this line. Since the baseline is moving, the vehicle will approximately describe a zigzag pattern along the river, sweeping the entire area. Fig. 3 shows three pieces of such trajectory, as well as the location of the baseline at three different times. Furthermore, the "period" of the trajectories with respect to the river length can be defined by the velocity of the boats.

The vehicle will navigate in LBL mode, measuring its distance to each beacon at a time, as described bellow. Although the vehicle does not navigate in a fixed frame, the absolute position of the beacons will be logged throughout the mission so that all measurements can be spatially located with respect to an earth fixed frame upon mission completion.

\section{ACOUSTIC NETWORK OPERATION}

Let $(x, y)$ be the coordinates of the vehicle in the moving frame associated with the acoustic beacons. The origin of this frame will be the position of beacon B1. The $x$ axis will be defined by the line joining the two beacons, positive from B1 to B2. The $y$ axis makes a clockwise 90 degrees angle with the $x$ axis. To avoid ambiguities in the determination of vehicle position, the vehicle will never cross the $x$ axis, meaning that its $y$ coordinate will never change sign. To simplify the presentation, this coordinate will be assumed positive.

The position of the vehicle can be computed from the distances of the vehicle to each of the beacons, $d_{1}$ and $d_{2}$, respectively, according to the following equations

$$
\begin{aligned}
& x=\frac{d_{1}^{2}-d_{2}^{2}+l^{2}}{2 l} \\
& y=\sqrt{d_{1}^{2}-x^{2}}
\end{aligned}
$$

where $l$ is the distance between the two beacons. Since this distance is required to determine the vehicle position, either

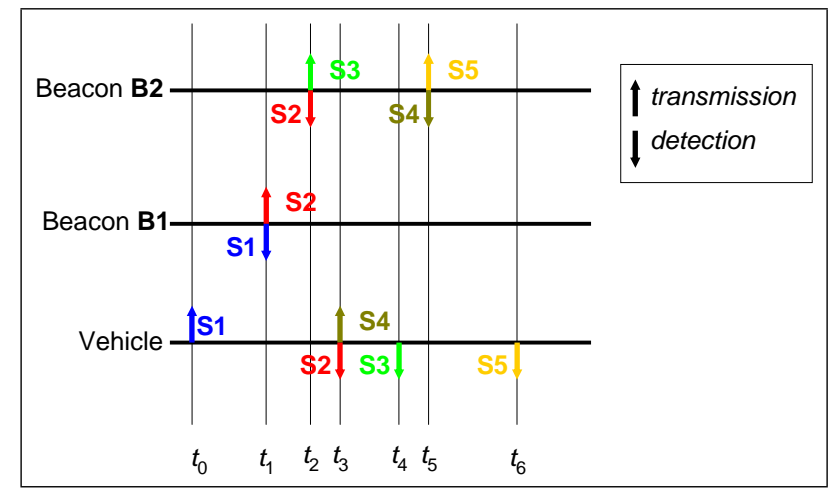

Fig. 4. Acoustic signal exchanges.

it is kept constant during the execution of a vehicle mission or it can change and the vehicle must determine its value in real time. The first possibility is difficult to implement since it requires a very accurate dynamic positioning of the beacons. We will consider, instead, the second possibility, for which additional acoustic signal exchanges are required for the vehicle to determine the distance between the beacons.

The acoustic network will operate using concepts both from spherical and from hyperbolic navigation techniques. In each localization cycle, the vehicle measures 3 different time intervals, allowing the determination of its distance to both beacons, as well as the distance between the beacons. A new cycle starts when the vehicle interrogates beacon B1 $\left(t=t_{0}\right)$, using signal $S_{1}$. Upon the detection of this signal, beacon B1 transmits the signal $S_{2}\left(t=t_{1}\right)$. The detection of signal $S_{2}$ by the vehicle allows the determination of the distance $d_{1}$ $\left(t=t_{3}\right)$. On the other hand, the beacon $\mathbf{B 2}$ will transmit the signal $S_{3}$ when it detects the signal $S_{2}\left(t=t_{2}\right)$. The detection of $S_{3}$ by the vehicle, when $t=t_{4}$, allows the determination of the distance corresponding to

$$
m=d_{1}+d_{2}+l .
$$

The second phase of the cycle starts when the vehicle detects $S_{2}$. At that moment, the vehicle interrogates beacon B2, using the signal $S_{4}$. This beacon will reply with the signal $S_{5}$, at $t=t_{5}$. The detection of this signal by the vehicle allows the determination of the distance $d_{2}$ and also of $l=m-d_{1}-$ $d_{2}$, completing the cycle. Fig. 4 shows the transmissions and detections of the different signals within a positioning cycle at $t=t_{6}$.

The values $d_{1}, m$ and $d_{2}$ are determined from the time intervals measured by the vehicle according to

$$
\begin{aligned}
d_{1} & =\frac{t_{3}-t_{0}}{2 c} \\
m & =\frac{t_{4}-t_{0}}{c} \\
d_{2} & =\frac{t_{6}-t_{3}}{2 c}
\end{aligned}
$$

where $c$ is the sound speed in the water.

If each new positioning cycle starts within a fixed amount of time after the completion of the previous one, the mechanism 


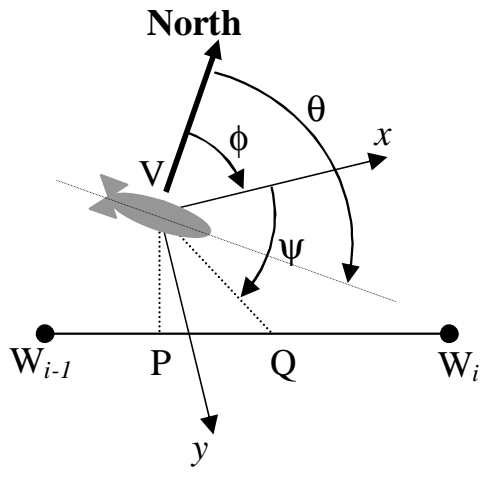

Fig. 5. Vehicle guidance.

described in [8] can be employed to externally track the vehicle evolution. It should be noted that the tracking solution will be computed also in the moving frame. However, absolute tracking can be easily implemented if each boat carries a GPS receiver and transmits in real time its position to the tracking station.

\section{Mission SPECIFICATION AND VEHICLE GUIDANCE}

The vehicle control system uses the current estimate of the vehicle position to make the vehicle describe the desired trajectory. If the acoustic network is moving, and taking into account that the vehicle does not receive data that allows the computation of its absolute position, the vehicle mission has to be specified in the moving frame associated with the beacons.

The vehicle mission is defined as a sequence of way-points $\left(x_{i}, y_{i}\right)$, all defined in the moving frame. The definition of these sequence of points should take into account the intended motion of the beacons, so that a realistic trajectory of the vehicle is obtained. Besides the definition of the way-points, the mission specification also includes the vehicle velocity and depth for the path connecting each pair of consecutive way-points. These values are directly used as references for the vehicle speed and depth controllers. The reference for the heading controller is computed by the guidance algorithm [12].

Fig. 5 shows the way the heading reference is computed. The current position of the vehicle, $\mathrm{V}$, is projected on the line joining the way-point $\mathrm{W}_{i-1}$ to the way-point $\mathrm{W}_{i}$, defining the point $\mathrm{P}$. Then, the point $\mathrm{Q}$ on the line joining $\mathrm{W}_{i-1}$ to $\mathrm{W}_{i}$ is defined in a way such that $\overline{\mathrm{W}_{i} \mathrm{Q}}=\alpha \overline{\mathrm{W}_{i} \mathrm{P}}$, where $\alpha \in[0,1]$ is a design parameter that balances accuracy of line tracking and control effort. The heading reference, $\psi$, is obtained from the direction of the line joining $\mathrm{V}$ to $\mathrm{Q}$.

Since the way-points and the position of the vehicle are defined in moving frame, all these computations have to be performed in this frame. Therefore, the computed heading reference, $\psi$, is defined with respect to the moving reference frame.

The heading of the vehicle, $\theta$, measured by the digital compass, is defined with respect to the north direction. Therefore, the heading controller reference must be defined with respect to the north direction too. Therefore, the reference for the heading controller will be $\psi+\phi$, where $\phi$ is the angle that the moving frame $x$ axis makes with the north direction. Although the motion of the acoustic beacons can be specified in a way that this orientation angle $\phi$ does not change too much during the execution of a mission, the vehicle navigation system needs to estimate this angle in real time.

\section{VEHICLE NAVIGATION}

For the estimation of the horizontal vehicle position in the frame associated with the moving beacons, the navigation algorithm fuses discrete distance measurements (ranges to the beacons, $d_{1}$ and $d_{2}$, and baseline length, $l$ ) together with continuous dead-reckoning data (velocity of the vehicle with respect to the water, $v$, and absolute vehicle heading, $\theta$ ). Besides the $(x, y)$ position of the vehicle, the navigation system also estimates the orientation of the moving frame, $\phi$.

The navigation algorithm is based on a discrete-continuous extended Kalman filter. The state of the filter is

$$
E=\left(x, y, \phi, c_{x}, c_{y}, l\right)
$$

where $(x, y)$ is the estimate of the vehicle position in the moving frame, $\phi$ is the estimate of the orientation of the fixed frame, $c_{x}$ and $c_{y}$ are estimates of the water current expressed in the moving frame and $l$ is the estimate of the baseline length.

The filter also keeps a matrix, $P$, of covariances of error estimates that is used to assess the quality of the estimation.

Between the instants of time when new range measurements are received, the evolution of the filter state, $E$, is governed by the following differential equation

$$
\dot{E}=F(E, v, \theta)=\left[\begin{array}{c}
v \cos (\theta-\phi)+c_{x} \\
v \sin (\theta-\phi)+c_{y} \\
0 \\
0 \\
0 \\
0
\end{array}\right]
$$

which is the deterministic part of the equation modeling the evolution of the estimated variables.

The covariance matrix evolves according to

$$
\dot{P}=\frac{\partial F}{\partial E} \cdot P+P \cdot \frac{\partial F}{\partial E}^{\mathrm{T}}+Q
$$

where $Q$ is a symmetric semi-positive definite matrix that represents the rate of increase of the error covariance due to the simplifications made in the state evolution equation.

Whenever a new distance measurement is available, the state $\mathrm{E}$ and the covariance matrix $\mathrm{P}$ are corrected according to the expressions

$$
\begin{aligned}
& E^{+}=E^{-}+K \cdot\left(z-z^{*}\right), \\
& P^{+}=P^{-}-K \cdot H \cdot P^{-} .
\end{aligned}
$$

where superscripts ${ }^{-}$and ${ }^{+}$denote values before and after the correction, respectively.

In these equations, $z$ stands for the distance measurement. Depending on the measurement type, it can be the range to 


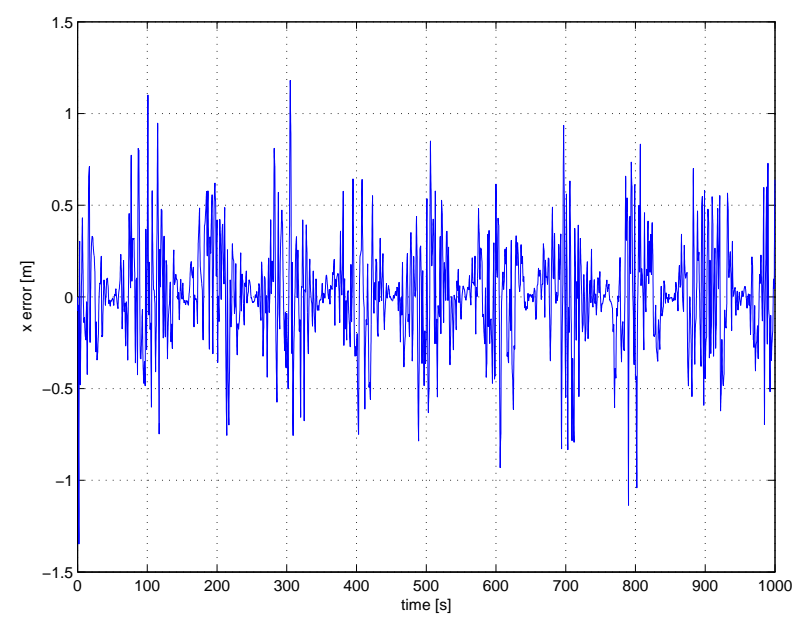

Fig. 6. Error in coordinate $x$.

beacon $\mathbf{B 1}\left(z=d_{1}\right)$, the range to beacon $\mathbf{B 2}\left(z=d_{2}\right)$, or the baseline length $(z=l)$. $z^{*}$ represents the expected range measurement, computed from the filter state variables, and equal to $\sqrt{x^{2}+y^{2}}$, to $\sqrt{(x-l)^{2}+y^{2}}$, or just to $l$, respectively. $H$ is the jacobian of the expected measurement with respect to the filter state, that is $H=\frac{\partial z^{*}}{\partial E}$, and is computed at the point $E^{-} . K$ is the Kalman gain given by

$$
K=P^{-} \cdot H^{\mathrm{T}} \cdot\left[H \cdot P^{-} \cdot H^{\mathrm{T}}+R\right]^{-1}
$$

where $R$ is the covariance of the error associated to the measurement.

\section{Simulation RESUlTS}

A simulator based on Matlab-Simulink was developed to evaluate the performance of the navigation algorithm. This section presents simulation results from an experiment showing the good performance of this algorithm.

In the experiment, the two beacons are located $100 \mathrm{~m}$ apart and move at a constant speed in a direction perpendicular to the baseline. The vehicle trajectory is a $100 \mathrm{~m}$ long straight line, parallel to the baseline at a distance of $50 \mathrm{~m}$. The vehicle just goes forth and back along this line at $1 \mathrm{~m} / \mathrm{s}$. Range measurements were corrupted by white noise with $1 \mathrm{~m}$ of standard deviation.

Figures 6 and 7 show the errors in the $x$ and $y$ coordinates of the moving frame of the position estimated by the navigation filter. These values have standard deviations of 0.25 $\mathrm{m}$ and $0.86 \mathrm{~m}$, respectively, showing that the filter is able to accurately estimate the position of the vehicle in the moving frame. Estimation errors are greater for the $y$ coordinate due to the geometric dilution of precision of the navigation solution [9].

Fig. 8 shows the evolution of the lengths of the major and minor axes of the uncertainty ellipse. The lengths are computed from the error covariance matrix, maintained by the Kalman filter based algorithm. The higher values occur when the vehicle is turning and are easily explained since the model

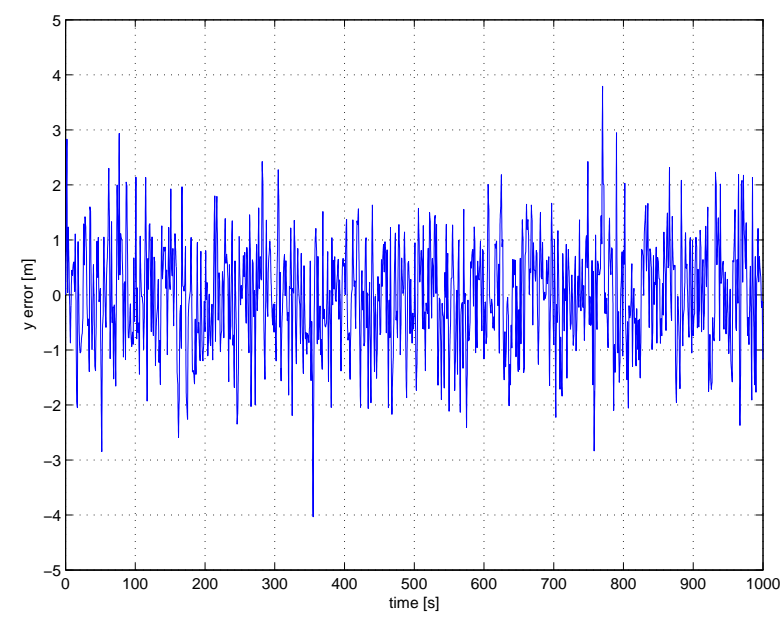

Fig. 7. Error in coordinate $y$.

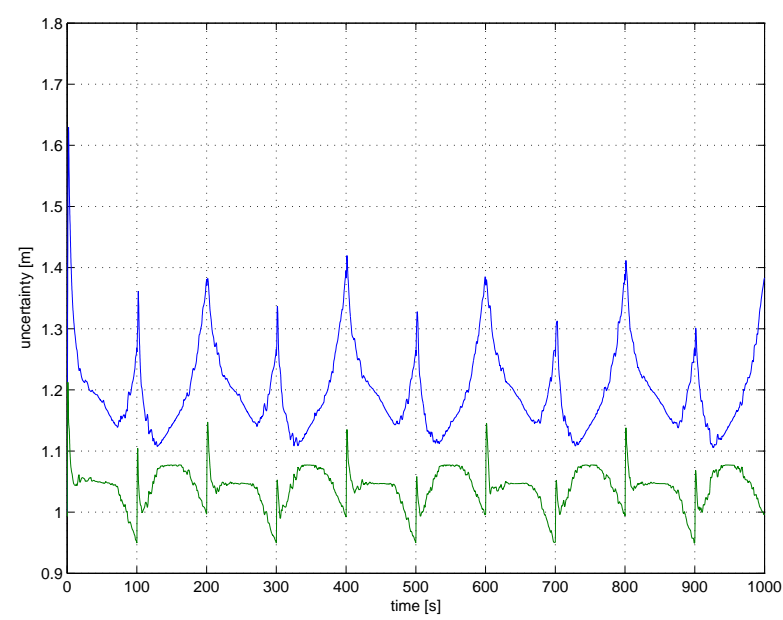

Fig. 8. Uncertainty ellipse axes.

of the vehicle motion is less accurate when the vehicle makes tight turns.

\section{COnClusions}

The operation mode of the acoustic network proposed in this paper allows for the navigation of an AUV when the network is moving. The additional signal exchanges required for the relative positioning do not increase the length of each positioning cycle, therefore assuring the same position accuracy of a fixed network.

The navigation algorithm described here is able to estimate in real time the orientation of the moving frame, a value that is required for the guidance of the AUV, since the mission is specified in the moving frame.

The simulation results already obtained, some of which are presented, show the effectiveness of the proposed solution. Currently, the authors are developing a system that will allow the field testing of this solution. 


\section{ACKNOWLEDGMENT}

This work was supported by the project SINS — Integrated system for navigation and tracking of multiple underwater vehicles - funded by the Electrical and Computer Engineering Department of Faculdade de Engenharia da Universidade do Porto (ref. DEEC-ID/X/2003), and by the project MUV - Multiple Underwater Vehicles - funded by FCT and Programa POSI (ref. POSI/SRI/47351/2002).

\section{REFERENCES}

[1] C. von Alt, B. Allen, T. Austin, and R. Stokey. Remote environmental measuring units. In Proc. of the IEEE Symp. on AUV Techn. AUV'94, Cambridge, MA, USA, July 1994.

[2] N. Cruz, A. Matos, A. Martins, J. Silva, D. Santos, D. Boutov, D. Ferreira, and F. Pereira. Estuarine environment studies with Isurus, a REMUS class AUV. In Proc. of the MTS/IEEE Conf. Oceans'99, Seattle, WA, USA, September 1999.

[3] P. Ramos, M. Neves, and F. Pereira. Using near field model predictions to detect and map an outfall sewage plume with an autonomous underwater vehicle. In Proc. of the MTS/IEEE Conf. Oceans'02, Biloxi, MI, USA, September 2002.

[4] Barbara Fletcher. Chemical plume mapping with the REMUS autonomous underwater vehicle. In Proc. of the Int. Symp. UUST'01, Durham, NH, USA, August 2001.
[5] J. Farrell, W. Li, S. Pang, and R. Arrieta. Chemical plume tracing experimental results with a REMUS AUV. In Proc. of the MTS/IEEE Conf. Oceans'03, San Diego, CA, USA, September 2003.

[6] Paul J. Ryan. Mine countermeasures a success. Naval Institute Proceedings, May 2003.

[7] Nuno Cruz, Aníbal Matos, and João Sousa. Small size AUVs: Operation results and new mission concepts. In Proceedings of the 5th IFAC Symposium on Intelligent Autonomous Vehicles IAV'2004, Lisbon, Portugal, July 2004.

[8] N. Cruz, L. Madureira, A. Matos, and F. Pereira. A versatile acoustic beacon for navigation and remote tracking of multiple underwater vehicles. In Proc. of the MTS/IEEE Conf. Oceans'01, Honolulu, HI, USA, November 2001.

[9] A. Matos, N. Cruz, A. Martins, and F. Pereira. Development and implementation of a low-cost LBL navigation systems for an AUV. In Proc. of the MTS/IEEE Conf. Oceans'99, Seattle, WA, USA, September 1999.

[10] A. Matos, N. Cruz, and F. L. Pereira. Post mission trajectory smoothing for the Isurus AUV. In Proc. of the MTS/IEEE Conf. Oceans'03, San Diego, CA, USA, September 2003.

[11] A. Matos and N. Cruz. Algorithms for external tracking of an AUV. In Proceedings of the 5th IFAC Symposium on Intelligent Autonomous Vehicles IAV'2004, Lisbon, Portugal, July 2004.

[12] J. Silva, A. Martins, and F. Pereira. A reconfigurable mission control system for underwater vehicles. In Proc. of the MTS/IEEE Conf. Oceans'99, Seattle, WA, USA, September 1999. 